Ayurlog: National Journal of Research in Ayurved Science

\title{
Review of Literature of Agnikumar rasa - A herbo-mineral ayurvedic formulation
}

\section{Kattewar Balaji Devrao ${ }^{1}$, Gangaprasad Asore², Gayatri Ashok Jagtap ${ }^{3}$}

1. Professor, Rasa shastra and bhaishajya kalpana,dhanvantari Ayurvedic Medical College, Udgir, Maharashtra.

2. Associate Professor, Rasa shastra and bhaishajya kalpana, APM's Ayurved Mahavidyalaya, Sion Mumbai-22, Maharashtra

3. P.G. Scholar Rasa shastra and bhaishajya kalpana, APM's Ayurved Mahavidyalaya, Sion, Mumbai-22, Maharashtra.

*Corresponding author: bdk73prof@gmail.com

\begin{abstract}
Rasashastra and bhaishajya kalpana is one of the branch of ayurveda that deals. with the pharmaceutical processing: preparation of compound medicines an therapeutic utility of herbal drugs, metals and minerals. Agnikumar Rasa is one such kharaliya herbo-mineral formulation mentioned in various Ayurvedic text. It is primarily used in the management of Agnimandya. According to Ayurveda, Agni has an important role inthe physiological functions of the body. Even there are reference to be used in disease such as Ajirna, Gulma, Visuchika, Grahani, etc. Amongst all formulations termed as Agnikumar rasa have been quoted by most seers and are well acclaimed in the management of agnimandya-janyaroga. Though variation is observed in the description of these kharaliya rasayana and compilation of these will help to study in comparative manner.
\end{abstract}

Keywords - Agnikumar rasa, kharaliya rasayan

\section{Introduction}

The word Rasashastra literally means the "Science of Mercury". However, it is a specialized branch of Ayurveda dealing mainly with materials which are known as 'Rasa dravyaas'. They have the following three characteristic attributes: instant effectiveness, requirement of very small doses and extensive therapeutic utility irrespective of constitutional variation. The following Sloka (verses normally in two lines) describes the above given attributes of rasa.

\section{"alpamaatropayogitwaat arucera} aprasangataha.

Kshipram aarogyadaayitwat aushadhyebhyo adhiko rasaha"

(Vaagbhata, Rasaratnasamcchay $28 / 1,1976)^{[1]}$

Thus, the above all characteristics are seen in kharaliya rasa which is combination of herbal drugs and metals-minerals.

Agnikumar rasa is one such kharaliya rasa used in the management of Agnimandya 
janya rogas. There are various references available having name as Agnikumar rasa in various Rasa aushadhis text. Though variation in ingredients is observed among these, there is resemblance in almost all formulations in perspective of levigation. Thus, present study is an attempt to go through references of Agnikumar rasa formulations in literature in order to have comparative better understanding of these formulations in terms of their composition, drugs specified for levigation, indications and contribution of various texts regarding this particular drug.

\section{Aim}

Aim of the present study is to compile all available references of Agnikumar rasa available in text and to study them in comparative manner in terms of their composition, method of preparation, dose, indications and contribution of various texts regarding these particular formulation.

\section{Materials and methods}

References of Agnikumar rasa will be compiled from various Rasa text and compendium andparticular contribution of each text will be noted.

\section{Literary review}

Agnikumar Rasa is not mere a single formulation but a variety of formulations across different texthave been quoted as Agnikumar Rasa. Name of drug itself throws light on the action of the compound i.e.it is a formulation which aids to cure the agnimandya janya disease (disease formed due to low digestive power). Earliest citation of Agnikumar Rasa is observed in rasa paddhati a book of 13th AD. Rasayogasagara compilatory book of Rasaushadhi for first time has compiled 4 formulations entitled as Agnikumar Rasa across different text. Following citations have been found in text such as Bhaishajya ratnavli, Yoga Ratnakar, Rasa Chandanshu and Rasaratnasamucchay. Other references have been collected from compendium Bharat bhaishajya ratnakar and are listed below:

Table no. 1 Reference of Agnikumar Rasa mentioned in Rasayogasagar ${ }^{[2]}$

\begin{tabular}{|l|l|l|l|l|}
\hline $\begin{array}{l}\text { Name of } \\
\text { ingredient }\end{array}$ & Rys 1 31 & Rys 2 23 & Rys 3 26 & Rys 4 27 \\
\hline Parad & 1 & 1 & 1 & 1 \\
\hline Gandhak & 1 & 1 & 1 & 1 \\
\hline Trikatu & & 2 & $1-1$ & 1 \\
\hline Bachnag & & & 1 & \\
\hline Tankan & & & & 1 \\
\hline 5 lavan & & & & Sai,vid \\
\hline Jeerak & & & & Jeearakdwaya \\
\hline Vidanga & 1 & Bhasma1 & & 1 \\
\hline Tamra & & 1 & & \\
\hline Hartal & & 1 & & \\
\hline Hingul & & 1 & & 1 \\
\hline Abhrak & 1 & & 1 \\
\hline Kharpar & & & & 1 \\
\hline Jaatifal & & & & 1 \\
\hline Lavang & & & \\
\hline Talispatra & & & \\
\hline
\end{tabular}




\begin{tabular}{|l|l|l|l|l|}
\hline Shrungi & & & & 1 \\
\hline Pippali & & & & 1 \\
\hline Yawani & & & & 1 \\
\hline Hingu & & & & 1 \\
\hline Jambeer & & & & $\mathrm{Rq}$ \\
\hline Chitrak & $\mathrm{Rq}$ & $\mathrm{Rq}$ & $\mathrm{Rq}$ & \\
\hline Bringa & 7 & Valukayantra & & \\
\hline Nimbuk & & & & \\
\hline Guduchi & 7 & & \\
\hline Trifala & Rqvalukayantra & & \\
\hline Suran & 7 & & & \\
\hline Nirgundi & 7 & & & \\
\hline
\end{tabular}

(Rys= Rasayogasagar)

Table no. 2. Reference of Agnikumar Rasa mentioned in Bhaishajyaratnavali ${ }^{[3]}$

\begin{tabular}{|c|c|c|c|}
\hline Name of ingredient & $\begin{array}{l}\text { B.r 5/528 } \\
\text { Jwar }\end{array}$ & B.r 10/115 agnimandya & $\begin{array}{l}\text { B.r 8/237 } \\
\text { Grahani }\end{array}$ \\
\hline Parad & & 1 & 1 \\
\hline Gandhak & & 1 & 1 \\
\hline Maricha & 1 & 8 & 1 \\
\hline Vacha & 1 & & \\
\hline Kushtha & 1 & 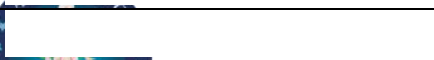 & \\
\hline Musta & 1 & & \\
\hline Bachnag & 4 & 3 & 1 \\
\hline Tankan & & 1 & 1 \\
\hline Kaparda & & 3 & \\
\hline Shankha & & 3 & \\
\hline Lohabhasma & & & 1 \\
\hline Magadhi & & & 1 \\
\hline Suntha & & & 1 \\
\hline \multicolumn{4}{|l|}{ Ajmoda } \\
\hline Jambeer & & 1 prahar & \\
\hline Aadrak & R.q. & & \\
\hline Abhrakbhasma & & & 10 \\
\hline Ahifen & & & R.q \\
\hline $\begin{array}{l}\text { Chitrak } \\
\text { Mool }\end{array}$ & & & R.q \\
\hline Matra & 1 Ratti & 1 Gunja & Marichaakar \\
\hline Anupan & Sunthijal & & Shitodak \\
\hline
\end{tabular}

(b.r= bhaishajyaratnavali) 
Table no. 3 References of Agnikumar Rasa mentioned in Rasendramangal ${ }^{[4]}$, Rasendrachintamani $^{[4]}$, Yogratnakar ${ }^{[5]}$

\begin{tabular}{|c|c|c|c|c|c|}
\hline $\begin{array}{ll}\text { Name } & \text { of } \\
\text { ingredient } & \end{array}$ & R.m 22 & R.chin1 & R.chin2 & Y.r1 30 & Y.r 225 \\
\hline Parad & 1 & $2 \mathrm{krsh}$ & 1 & 1 & 1 \\
\hline Gandhak & 1 & $2 "$ & 1 & 1 & 1 \\
\hline Maricha & & & 10 & 1 & 8 \\
\hline Bachnag & & & 3 & & 1 \\
\hline Tankan & & & 1 & & 1 \\
\hline Kaparda & & & 1 & 2 & 2 \\
\hline Shankha & & & & 2 & 2 \\
\hline Sajjikhar & & & 1 & & \\
\hline Magadhi & & & 1 & & \\
\hline Suntha & & & 1 & & \\
\hline Nag & 1 & & & & \\
\hline Jambeer & & & $\mathrm{Rq}$ & 7 & \\
\hline \multicolumn{6}{|l|}{ Aadrak } \\
\hline Hansapadi & $1 \mathrm{D}$ & Kupi 1 din & & & \\
\hline $\begin{array}{l}\text { Kanta } \\
\text { Karifala }\end{array}$ & Kachkupi for $12 \mathrm{hrs}$ & & & & \\
\hline Vijaya & 1/6th of bachnag & & & & \\
\hline Shigru & $1 / 2$ part of marich & & & & \\
\hline Nimbuk & & & & 3 times $1 \mathrm{D}$ & \\
\hline
\end{tabular}

(R.m=Rasendramangal,R.chin=Rasendrachintamani,Y.r=Yogratnakar)

Table no. 4 Reference of Agnikumar Rasa mentioned in Rasaratnasamucchaya ${ }^{[6]}$

\begin{tabular}{|l|l|l|l|l|l|}
\hline Name of ingredient & R.r.s1 & R.r.s2 13 & R.r.s3 14 & R.r.s4 15 & R.r.s5 \\
\hline Parad & 1 & & 1 & 1 & 1 \\
\hline Gandhak & 1 & 1 & 1 & 1 & \\
\hline Jaipal & 1 & & & & \\
\hline Trifala & 1 & & & & \\
\hline Trikatu & 1 & & & $1-1$ & \\
\hline Bachnag & & 1 shan & & 1 & 1 \\
\hline Tankan & & & & 1 & \\
\hline Kaparda & & & & 1 & \\
\hline
\end{tabular}




\begin{tabular}{|l|l|l|l|l|l|}
\hline Jambeer & & & & $\mathrm{Rq}$ & \\
\hline Aadrak & & & $\mathrm{Rq}$ & & \\
\hline Gomutra & 16 & & & & 10 tola \\
\hline Hansapadi & & 1 & $3 \mathrm{D}$ & & $\begin{array}{l}\text { Bachnag-goli } \\
\text { above- } \\
\text { bachnag }\end{array}$ \\
\hline Kantakarifala & & Valukayantra & & & $\begin{array}{l}\text { Valukayantra - } \\
1.5 \text { days }\end{array}$ \\
\hline
\end{tabular}

(R.r.s=Rasaratnasamucchaya)

Table no. 5 References of Agnikumar Rasa mentioned in Rasa ratnakar ${ }^{[3]}$

\begin{tabular}{|c|c|c|c|c|c|}
\hline Name of ingredient & R.r1 6 & R.r2 7 & R.r3 8 & R.r4 9 & $\begin{array}{l}\text { R.r5 } \\
10\end{array}$ \\
\hline Parad & 1 & 1 & 1 & 1 & 1 \\
\hline Gandhak & 1 & 1 & 1 & 1 & \\
\hline Trifala & & & 1 & & \\
\hline Trikatu & & & 1 & & \\
\hline Maricha & 3 & & & & 1 \\
\hline Vacha & & & & & 1 \\
\hline Bachnag & 1 & 1 & 1 & & \\
\hline Tankan & & & & 2 & \\
\hline Suntha & & & & & 1 \\
\hline 2 kshar & & $1-1$ & $1-1$ & & \\
\hline 5 lavan & & $1-1$ & Sss & & \\
\hline Shigrumool & & 2.5 & & & \\
\hline Vijaya & & 5 & & & \\
\hline Ajmoda & & & 1 & & \\
\hline Chitrak & & & 1 & & \\
\hline Jeerak & & & 1 & & 1 \\
\hline Vidanga & & & 1 & & \\
\hline
\end{tabular}




\begin{tabular}{|c|c|c|c|c|c|}
\hline Kuchala & & & 18 & & \\
\hline Dhatri & & & & 1 & \\
\hline Hingu & & & & & 1 \\
\hline Jambeer & & & Rq. & Rq. & \\
\hline Aadrak & & & Marichabha & Varatikagajaput & \\
\hline $\begin{array}{l}\text { Kanta } \\
\text { Karifala }\end{array}$ & 21 & & & & \\
\hline Vijaya & & 1 & & & \\
\hline Shigru & & 1 & & & \\
\hline Chitrak & & 1 & & & \\
\hline Bringa & & 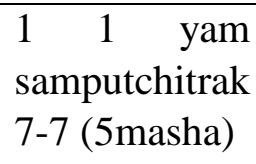 & & & $\mathrm{Rq}$ \\
\hline Nimbuk & & & & & $\mathrm{Rq}$ \\
\hline
\end{tabular}

(R.r=Rasa ratnakar)

Table no. 6 References of Agnikumar rasd mentioned in Rasajsundar $^{[3]}$

\begin{tabular}{|l|l|l|}
\hline Name of ingredient & R.ras1 25 & R.ra.s 2 28 \\
\hline Parad & 1 & 1 \\
\hline Gandhak & 1 & 1 \\
\hline Maricha & 8 & \\
\hline Bachnag & 1 & 1 \\
\hline Tankan & 1 & \\
\hline Kaparda & 2 & \\
\hline Shankha & 2 & Rq \\
\hline Hansapadi & & $\begin{array}{l}\text { Valukayantra 3 yama } \\
1 / 2 \text { part bachnag f/b }\end{array}$ \\
\hline $\begin{array}{l}\text { Kanta } \\
\text { Karifala }\end{array}$ & & \\
\hline Vijaya & & Trikatu+saindhav+aadrak \\
\hline Shigru & & Rq \\
\hline Chitrak & & \\
\hline
\end{tabular}

(R.ra.s=Rasarajsundar)

Table no. 7 References of Agnikumar Rasa mentioned in Rasa Chandanshu ${ }^{[7]}$

\begin{tabular}{|l|l|l|l|l|}
\hline Name of ingredient & R. chan 1 & R. chan 2 & R. chan3 30 & R. chan2 29 \\
\hline
\end{tabular}




\begin{tabular}{|c|c|c|c|c|}
\hline Parad & 1 & 1 & 1 & 2 \\
\hline Gandhak & 1 & 1 & 1 & 1 \\
\hline Jaipal & 1 & & & 1 \\
\hline Trifala & 1 & & & 3 \\
\hline Trikatu & 1 & 1 & & 1 \\
\hline Maricha & & & 1 & \\
\hline Kaparda & & & 2 & \\
\hline Shankha & & & 2 & \\
\hline 5 lavan & & $1-1$ & & $1-1$ \\
\hline Vijaya & & 10 & & \\
\hline Aadrak & & & & $\mathrm{Rq}$ \\
\hline Gomutra & 16 & & & \\
\hline Nimbuk & & & 3 TIMES 1D & \\
\hline Vijaya & & $3-3$ & & \\
\hline \multicolumn{5}{|l|}{ Chitrak } \\
\hline \multicolumn{5}{|l|}{ Bhringraj } \\
\hline Aadrak & & Bhavana & & \\
\hline
\end{tabular}

(R. chan=Rasa Chandanshu)

\section{Discussion:}

Total 31 reference have been mentioned in Rasa Aushadhi compendium and text. Observing closely to each reference suggest that each kalpa contains Dipan, Pachan and Agnivardhan dravyas. All the references given in the text shows that they mainly acts on Annavaha strotasa. The commonly present dravya Maricha which does pachan karma. Shankha bhasma is Uttam Deepan, Paachan dravya and Amlapittanaashak kalpa. Kapardik Bhasma is Ushna, Deepan karya does the action of Vaatanuloman and thus, used in Grahani disease. Bachnaag is toxic drug but when used after purification acts as Vyavayi, Vikasi, Kledashoshak and Aampaachak. Tankan is a Kshaariya dravya which acts as Aampaachak. Kajjali here acts as preservative to increase shelf life of this drug and even act as catalyst. Thus, Agnikumar rasa will help in the management of Agnimandya janya vikar such as Grahani, Gulma, Ajirna, Vishuchika, etc.

\section{Conclusion:}

The main root cause of disease is Agnimandya i.e indigestion or low digestive power as mentioned by Acharya 
Charak $^{[8]}$. So, while treating any agnimandya janya disease correction of Agni is needed. It is clear from the literature that Agnikumar rasa has been used to treat wide range of diseases of Gasto intestinal tract, abdominal conditions such as Diarrhoea, Cholera, Irritable bowel Syndrome, etc. Thus it has broad spectrum activity in the management of Agnimandya janya disorders.

\section{References}

1. Vagbhat, Rasaratnasamuchchaya. Shastri A (editor).9th ed. Chaoukhamba amarabharati publication,Varanasi; 1976.

2. Vd.Hariprapannaji, Rasayogasagar, Part 1, Yakaradi Rasa-430, Reprint edition, Chaukhambha krishnadas acadamy, Varanasi, 2004.

3. Govindadas, bhaishajyaratnavali. Mishra S (editor), choukhamba surabharati prakashan; Varanasi, Reprint 2007.

4. Shri Nagindas Chaganlal Shah Rasavaidya, Bharat bhaishajya ratnakar, B. Jain Publishers ltd. ,New delhi, Reprint 2012, Part 1.

5. Vd. Laxmipatisastri, Commenter, yogaratnakara, jwaradhikara, Reprint edition, Chaukhambha prakashana, Varanasi,2002

6. Vagbhat, Rasaratnasamuchchaya. Shastri A (editor).9th ed. Chaoukhamba amarabharati publication,Varanasi; 2006 6/Pg no. 183.

7. Vd.datto ballal borkar, Rasa chandanshu, $3^{\text {rd }}$ edition, Shri Gajanan book depot, Mumbai, Pg no.194,217, 356.

8. Agnivesha, Charak Samhita, 4th edi. Vidyotini hindi, commentary by Shastri K, Chikitsasthana, Chaukhamba, Sanskrit Sansthan,Varanasi, 1994.

Cite article:

Review of Literature of Agnikumar rasa - A herbo-mineral ayurvedic formulation Kattewar Balaji Devrao, Gangaprasad Asore, Gayatri Ashok Jagtap Ayurlog: National Journal of Research in Ayurved Science- 2019; (7)(1): 1-8 